\title{
Hypericum Oil
}

National Cancer Institute

\section{Source}

National Cancer Institute. Hypericum Oil. NCI Thesaurus. Code C71872.

An oil obtained by infusing hypericum flowers into a carrier oil. Hypericum oil has antiseptic and analgesic properties. 